\title{
EL ROL DE LA USABILIDAD PERCIBIDA SOBRE LA CONFIANZA EN LAS SUBASTAS ONLINE
}

O PAPEL DA USABILIDADE PERCEBIDA NA CONFIANÇA DE LEILÕES ONLINE

THE ROLE OF USABILITY AS PERCEIVED IN THE CONFIDENCE SHOWN IN ONLINE AUCTIONS

\section{RESUMEN}

Si bien se ha considerado que la usabilidad del sitio web es una de las variables clave en la formación de la confianza de los usuarios, esta relación no ha sido profundamente analizada respecto a su efecto directo o mediado. Dentro de este contexto, se propone que la usabilidad influye sobre la confianza, pero no de manera directa, sino más bien a través de la satisfacción, con esta última actuando como variable mediadora. El presente estudio se lleva a cabo en el caso particular de los sitios de subastas por Internet, que representan un mercado creciente y en auge entre los servicios a través de Internet en Latinoamérica. Luego de probar la unidimensionalidad, fiabilidad y validez de las escalas de medida, se contrastan las hipótesis a través de un modelo de ecuaciones estructurales y una estrategia de modelos rivales.

PALABRAS CLAVE Usabilidad, confianza, subastas por internet, remates online, satisfacción.

Eduardo Torres-Moraga eduardot@unegocios.cl
Profesor Asistente, Escuela de Negocios, Universidad de Chile - Santiago - Chile

Cristobal Barra cbarra@fen.uchile.cl

Instructor, Escuela de Negocios, Universidad de Chile - Santiago - Chile

Resumo Embora seja considerado que a usabilidade do website é uma das variáveis-chave na formação da confiança dos usuários, esta relação não tem sido profundamente analisada no que diz respeito ao seu efeito direto ou mediado. Dentro deste contexto, propõe-se que a usabilidade influi sobre a confiança, porém não de maneira direta, mas sim através da satisfação, com esta última atuando como variável mediadora. 0 presente estudo foi realizado no caso específico de websites de leilões, que representam um mercado em crescimento e que se encontram no auge entre os serviços oferecidos por meio da Internet na América Latina. Depois de testar a unidimensionalidade, confiabilidade e validade das escalas de medida, foram contrastadas as hipóteses por um modelo de equações estruturais e uma estratégia de modelos rivais

Palavras-Chave Usabilidade, confiança, leilões por Internet, arremates online, satisfação.

Abstract Usability has been considered as a key variable that allows trust development in online environments. However, the relationship between these concepts has not been studied in depth to realize if the effect is direct or mediated. In this context, it is proposed that the perceived usability affects trust not in a direct way but considering the role of satisfaction as a mediator. This study considers the specific context of Internet auctions that represents a growing services market through Internet in Latin America. After testing the unidimensionality, reliability and validity of the measurement scales, hypotheses were tested using a structural equation modeling, a rival models strategy and a multi-sample analysis.

keywords Usability, trust, internet auctions, online auctions, satisfaction. 


\section{INTRODUCCIÓN}

A fines de la década del 90, los sistemas de subastas (o remates) online generaron una revolución en la forma de realizar transacciones a través de Internet, permitiendo que las distancias y fronteras geográficas ya no sean un impedimento para que una persona se transforme en un comprador o vendedor de bienes y servicios. De hecho, este tipo de subastas se ha convertido en una real alternativa al comercio tradicional por tener la ventaja de permitir acceder de forma ágil, expedita y formal a la oferta de miles de productos.

Como en otras áreas del comercio electrónico, la confianza cumple un rol fundamental en el éxito de éstos sitios web. Incluso, cobra mayor relevancia por el hecho que las transacciones se llevan a cabo con una persona que es desconocida. Por esta razón, los sitios de remates por Internet han centrado sus esfuerzos en desarrollar procedimientos para que las transacciones se realicen de manera más confiable (FAN, TAN y WHINSTON, 2005), de tal manera que neutralicen cualquier intención de comportamiento oportunista por parte de los participantes (HU y otros, 2004). En concreto, han implementado por ejemplo, los servicios anti-fraude (débito online y servicios de garantía) (ANTONY, LIN y XU, 2006) y los sistemas de información dirigidos a dar a conocer la reputación de los usuarios (RESNICK y otros, 2000). Estos últimos, son los que han cobrado mayor relevancia (KOUFARIS y HAMPTON-SOSA, 2004), debido a que, como se ha podido constatar, afectan el precio final del producto e incluso el éxito de la transacción (MELNIK y ALM, 2002).

Pero además de estos mecanismos, existen indicios de que la usabilidad percibida podría ser un factor relevante de considerar en el momento de aumentar la confianza hacia los sitios de subastas por Internet. Esta idea se fundamenta en el hecho que la usabilidad percibida ha sido reconocida, en algunos estudios realizados en otros contextos online, como un factor relevante para la formación de confianza (FLAVIAN, GUINALIU y GURREA, 2006).

Conscientes de que estas relaciones no se han estudiado en este contexto específico, el presente estudio analiza empíricamente el rol que cumple la usabilidad percibida en la generación de la confianza hacia los sitios web de subastas. Considerando que la usabilidad percibida es un reconocido componente de la calidad del servicio online, en el presente estudio se analiza el rol que cumple la satisfacción como variable me- diadora en esta relación. Para cumplir este propósito, a continuación se realiza un análisis de la literatura que soporta el planteamiento de las hipótesis. Luego, en la metodología, se presentan los instrumentos de medición, los datos de la muestra y los análisis de unidimensionalidad, fiabilidad y validez de los constructos utilizados. Posteriormente, se contrasta el modelo teórico por medio de ecuaciones estructurales y se compara con diferentes modelos rivales que permiten contrastar la hipótesis expuesta en este estudio. Al final del artículo se presentan las conclusiones y la discusión de los resultados.

\section{ANTECEDENTES}

Los sitios de subastas online se pueden clasificar, junto a los foros y a las salas de chat, en la modalidad de C2C (consumer to consumer), es decir, como aquellas interacciones o transacciones vía Internet que se generan entre dos personas o usuarios finales (JONES y LEONARD, 2008).

Por sus características, las transacciones realizadas a través de los sitios de subastas online presentan mayores niveles de incertidumbre que las realizadas a través de los sistemas convencionales (CHEEMA y otros, 2005; DEWALLY y EDERINGTON, 2006). Esto se debe a la naturaleza interpersonal de las transacciones y a la asimetría de información que se presenta entre los compradores y vendedores que participan en las subastas (ANTONY, LIN y XU, 2006). Normalmente el envío de los productos ocurre una vez que se ha realizado el pago (LUCKING-REILEY, 2000), siendo muy difícil para el comprador revisar el producto antes de la compra. Además de lo anterior, no existe un respaldo tangible del cumplimiento de la contraparte (LI, SRINIVASAN y SUN, 2009), por lo que los compradores están obligados a asumir algunos riesgos adicionales, situándolos en una posición de desventaja frente al vendedor.

El funcionamiento de los portales de remates online se basa fundamentalmente en la inscripción de los usuarios en los registros del sitio web, proveyendo algún tipo de identificación única del país de origen (ID de identidad nacional) y una posterior verificación de datos (control que eventualmente podría no realizarse). Estos usuarios tienen la posibilidad de actuar como compradores o vendedores de los artículos (que se clasifican en categorías dentro de los sitios web), uti- 
lizando la plataforma del proveedor en un contexto de relativa confidencialidad (principalmente para evitar que una vez realizado el contacto, las transacciones se realicen por medio de otro canal). Así, las compañías de subastas online actúan como mediadores de la transacción, no asumiendo responsabilidad por los productos ofrecidos, ni por la información o los procesos de venta que realizan sus usuarios (LI, SRINIVASAN y SUN, 2009).

En Latinoamérica, pese a la existencia de algunos proveedores locales en cada uno de los países de la zona, la oferta de servicio de subastas online estuvo dominada por dos grandes proveedores, como son Mercado Libre y DeRemate. Desde Agosto de 2008, cuando adquirió a DeRemate, Mercado Libre se transformó en el líder indiscutido del mercado latinoamericano, con operaciones en 12 países y con una estructura de propiedad que incluye a grandes grupos económicos a nivel mundial. Lo anterior, ha generado una red de uso compartido entre los distintos países de Latinoamérica, permitiendo aumentar progresivamente el nivel de transacciones en la región.

\section{Confianza}

Se ha considerado la confianza en la literatura académica como uno de los constructos claves en el marketing relacional (DONEY y CANNON, 1997). Una definición que se ha aceptado en el contexto del comercio electrónico (ROY, DEWIT y AUBERT, 2001; JONES y LEONARD, 2008) señala que la confianza "es la buena voluntad de una parte, de ser vulnerable a las acciones de otra parte, basado en la esperanza de que la otra parte desempeñará una acción importante para quien confía, sin importar la habilidad de monitoreo o control sobre esa otra parte" (MAYER, DAVIS y SHOORMAN, 1995, p.712, traducción nuestra).

A pesar que la confianza se manifiesta de manera similar tanto en el contexto convencional como online (KRACHER, CORRITONE y WIEDENBECK, 2005), se ha reconocido que tiene una relevancia mucho mayor en un ambiente online. Esto se debe a que las transacciones se desarrollan en un medio más incierto, donde existe una distancia física, desconocimiento entre las partes y falta de contacto con una interfaz humana (URBAN, SULTAN y QUALLS, 2000; PAVLOU, 2003).

Considerando esto último, en la literatura se ha acuñado el concepto $e$-trust para referirse a la confianza depositada en los proveedores de servicios por Internet. En concreto, ésta se considera como una creencia que permite a los consumidores ser vulnerables a los proveedores de Internet, luego de tomar en cuenta las características de estos últimos. Esta e-trust, surge como un medio para asumir y disminuir el riesgo percibido (PAVLOU, 2003), influyendo en el comportamiento y en la lealtad de las personas hacia un determinado sitio web (CASALO, FLAVIAN y GUINALIU, 2007).

La confianza se hace necesaria en este contexto de mayor incertidumbre, permitiendo hacer frente a situaciones de riesgo donde los contratos y garantías están ausentes o son complejos de realizar (GRAZIOLI y JARVENPAA, 2000), y donde además, se genera un escenario ideal para que aquellas personas oportunistas puedan utilizar las asimetrías de información para generar situaciones de provecho frente a su contraparte (HU y otros, 2004).

\section{Usabilidad Percibida}

Debido a los notables cambios que han experimentado las tecnologías de información y sobre todo al crecimiento exponencial que ha tenido Internet estos últimos años, los estudios sobre usabilidad se han centrado fundamentalmente en los servicios vía web (AGARWAL y VENKATESH, 2002). Ante estos cambios, que incluyen un aumento en términos de cantidad, diversidad y roles de los usuarios, la usabilidad pasó de ser un concepto netamente técnico a uno más amplio que incluye la interacción y las percepciones de los usuarios respecto a la plataforma que utilizan (BARNES y VIDGEN, 2002).

Actualmente, diversos autores conceptualizan la usabilidad desde una perspectiva aplicada, considerándola como el esfuerzo requerido por parte de un usuario para utilizar un sistema computacional (CASALO, FLAVIAN y GUINALIU, 2007) o como un atributo componente de la calidad que permite al usuario el fácil manejo de un sistema (NIELSEN, 2003).

Se ha considerado que la usabilidad es un factor clave para el éxito de las empresas que operan a través de Internet (PALMER, 2002). De hecho, un buen nivel de usabilidad es una herramienta efectiva que permite sobrellevar uno de los problemas básicos que se presentan en los ambientes online, que es la falta de un empleado o persona con quien interactuar por parte del proveedor (LOONAM y O'LOUGHLIN, 2008). Además, permite reducir los errores, aumentar la precisión en la navegación, incrementar el uso y proveer una mejor percepción de la imagen gráfica del sitio web (NIELSEN, 2000). 
En el caso particular de los sitios web de subastas, debido a que la interacción entre el cliente y el proveedor se realiza online, la usabilidad percibida cobra una especial relevancia, transformándose en una herramienta fundamental para que el usuario tangibilice al proveedor y los servicios que se ofrecen a través de la web (FOGG y otros, 2002). Además, como los sistemas de navegación, inscripción y compra son más complejos que en otros servicios web, es de vital importancia lograr un fácil entendimiento y familiarización con las opciones y servicios ofrecidos en el portal. Desde esta perspectiva, la medición de la usabilidad se debería basar en la experiencia de uso por parte del cliente, enfocándose en la interface y en el entendimiento de la web (ZVIRAN, GLEZER y AVNI, 2006).

\section{Relación usabilidad-confianza}

Una de las formas más efectivas de reducir la incertidumbre es entregando información de una manera clara y precisa, de esta forma, un buen nivel de usabilidad es un medio para cumplir este objetivo en el contexto de las relaciones online (AGRIAWAN y THAKUR, 2008).

Son múltiples los antecedentes que señalan la existencia de una relación directa de influencia de la usabilidad sobre la confianza en ambientes basados en Internet. En este marco, Koufaris y Hampton-Sosa (2004) encontraron un efecto significativo entre estas variables en las etapas iniciales de formación de confianza. También, en un contexto más amplio, no especificando el tiempo de la relación, otros estudios han demostrado que esta relación es significativa y directa (KIM y AHN, 2007; AGRIAWAN y THAKUR, 2008).

Además, la facilidad de uso, que es comúnmente utilizada como sinónimo de la usabilidad percibida al ser uno de sus componentes más prominentes, se ha considerado como uno de los aspectos más importantes de la apariencia del sitio web, y consecuentemente como una señal de preocupación para ayudar a los usuarios a entender el funcionamiento de los sistemas online, generando posteriormente confianza hacia esos sistemas (GEFEN, KARAHANNA y STRAUB, 2003). En la misma línea, estudios más recientes, han reconocido la relación directa que existe en la facilidad de uso para lograr la confianza tanto en los ambientes tradicionales basados en Internet (TUNG y otros, 2009), como en los sitios especialmente diseñados para accederse por medio de dispositivos móviles (LI y YEH, 2010).

\section{FORMULACIÓN DE HIPÓTESIS}

La usabilidad percibida se ha considerado reiteradamente como un componente de la calidad del servicio online (BARNES y VIDGEN, 2002; YANG y FANG, 2004; LOONAM y O'LOUGHLIN, 2008). La calidad del servicio, a su vez, se relaciona de manera directa con la satisfacción, siendo uno de sus principales determinantes, incluso en las relaciones basadas en el uso de sitios Web (ZBOJA y VOORHES, 2006). De esta forma, se espera que los componentes de la calidad del servicio posean también una relación directa con la satisfacción y que de esta forma puedan afectar a constructos que son determinados por ésta.

Es relevante señalar que la relación usabilidadconfianza ha sido determinada como positiva y directa en contextos que carecen de la satisfacción como variable presente en el modelo, de esta forma, cobra relevancia estudiar el potencial rol mediador de ésta en la relación antedicha.

\section{Satisfacción como mediador}

En términos generales, la satisfacción es un estado afectivo del consumidor producto de la evolución global de su relación con un determinado proveedor (SANZO y otros, 2003). Esta evaluación global es el resultado de una comparación entre las expectativas del consumidor, antes del uso de un producto o servicio, y el desempeño efectivo en la entrega del servicio, que se evalúa con posterioridad (OLIVER, 1999). En concreto, la satisfacción es la consecuencia de la valoración de una brecha entre dos estímulos: el resultado y el referente de comparación (OLIVER, 1997). En esta misma línea, Szymanski y Hise (2000) consideran que la e-satisfaction es una comparación realizada por el usuario en la que intervienen, por una parte, la experiencia con los servicios ofrecidos a través de Internet, y por otra, la experiencia que ha tenido con el comercio tradicional.

Dentro de las principales ventajas de la satisfacción, es que actúa como un determinante de otros constructos que son muy relevantes dentro del contexto del marketing relacional, como son por ejemplo, el compromiso (DIMITRIADES, 2006), la reputación (SELNES, 1993) y como se presenta posteriormente, de la confianza. Estas ventajas, también se encuentran presentes en el contexto del comercio electrónico (EVANSCHITZKY y otros, 2004).

Precisamente en este contexto se ha planteado que 
la usabilidad percibida está fuertemente asociada a la satisfacción del usuario (PALMER, 2002). De hecho, altos niveles de usabilidad favorecen la comprensión de los contenidos y de las tareas disponibles en el sitio web, influyendo positivamente en el comportamiento futuro de los usuarios (CASALO, FLAVIAN y GUINALIU, 2007). La relación usabilidad-satisfacción ha sido respaldada y reconocida en diversos estudios (SPILLER y LOHSE, 1998; FLAVIAN, GUINALIU y GURREA, 2006). Entre estos estudios se puede destacar el realizado por Fu y Salvendy (2002), quienes determinaron que la usabilidad es el principal factor que contribuye a la satisfacción, tanto en la navegación a través de la web, como en la búsqueda de información en Internet. Además se puede mencionar el estudio de Zviran, Glezer y Avni (2006), quienes señalaron que una mejor interfaz de la web, tiene un mejor calce con los deseos y preferencias de los usuarios, lo que aumenta su satisfacción.

Por su parte, se considera que la satisfacción es uno los principales antecedentes de la confianza, ya que reduce la incertidumbre y el riesgo de optar por alternativas no conocidas por el consumidor (RAVALD y GRONROOS, 1996). Una experiencia satisfactoria con el proveedor aumenta los niveles de confianza y la tendencia a continuar utilizando el producto o servicio (KIM, FERRIN y RAO, 2003). Si bien, la mayoría de los estudios han sido aplicados al contexto de las marcas dentro del comercio tradicional (ZBOJA y VOORHES, 2006), en el contexto del comercio electrónico, diversas investigaciones han confirmado la existencia de esta relación. Es así como, por ejemplo, los estudios de Walczuch y Lundgren (2004) y Pavlou (2003) han comprobado que la satisfacción genera un efecto positivo sobre la confianza del usuario en el contexto de los servicios online y de los e-tailer respectivamente.

Considerando que existe una secuencia lógica en las relaciones de usabilidad-satisfacción y satisfacciónconfianza, la relación entre usabilidad y confianza podría no ser directa, como lo indican algunos estudios previos (ROY, DEWIT y AUBERT, 2001), sino más bien indirecta y mediada por la satisfacción, de esta forma es posible plantear la siguiente hipótesis, ampliando su dominio al contexto de los sitios de subastas online:

H: La relación de influencia de la usabilidad percibida por los usuarios sobre la confianza hacia los portales de subastas online no es directa, sino indirecta y mediada por la satisfacción.

\section{METODOLOGÍA}

Sobre la base de la literatura especializada en la materia, la consulta de expertos y test previos realizados a los usuarios de páginas web de remates en Internet, se construyeron escalas de medida para los conceptos de usabilidad percibida, satisfacción y confianza, asegurando de esta manera su validez de contenido. En concreto, para desarrollar la escala de confianza se consideraron los trabajos de Kumar, Scheer y Steenkamp (1995), Doney y Cannon (1997) y Roy, Dewit y Aubert (2001), para usabilidad, las escalas de Roy, Dewit y Aubert (2001), Kim, Xu y Koh (2004) y Flavian, Guinaliu y Gurrea (2006) y para satisfacción, las escalas de Brockman (1998), Sharma y Patterson (2000) y Veloutsou, Daskou y Daskou (2004).

Posteriormente estas escalas fueron depuradas (DE WULF y ODEKERKEN-SCHRODER, 2003), realizando 4 focus groups de usuarios de los servicios de subastas online y entrevistas con 4 ejecutivos de diferentes empresas de comercio electrónico y de subastas online. En los focus groups participaron un total de 28 personas, de las cuales el 64\% fueron hombres, el 56\% eran profesionales y ejecutivos, el $21 \%$ realizaban un trabajo técnico o manual, $17 \%$ estudiaban y el $7 \%$ en ese momento estaba en busca de trabajo. Además, el 65\% tenía educación universitaria, el 25\% educación técnico profesional y el 10\% educación secundaria. Considerando que estas escalas fueron construidas a partir de estudios realizados en otros contextos, tanto los focus groups como las entrevistas, tuvieron como objetivo readecuar y/o eliminar aquellos indicadores más conflictivos o redundantes, definiendo aquellos que mejor reflejan la confianza, la satisfacción y la usabilidad percibida en este contexto de estudio (ZAICHKOWSKY, 1985; LICHTENSTEIN, NETEMEYER y BURTON, 1990). Con estos análisis se obtuvieron las escalas con las cuales se construyó el cuestionario. Las afirmaciones, debían ser respondidas por medio de una escala Likert de 7 intervalos (Quadro 1).

El cuestionario final se aplicó por teléfono durante el mes de julio de 2008 en la ciudad de Santiago de Chile, a personas que alguna vez habían realizado algún tipo de transacción a través de un sitio web de subastas. Para este procedimiento se empleó el método de entrevistas CATI (computer aided telephone interview), que permite seleccionar de manera aleatoria el número telefónico a contactar (desde una base de datos que contiene el $70 \%$ de los números telefónicos del país) y el orden de las preguntas Likert presentadas a los entrevistados. Sobre un total de 6.300 personas 


\section{Quadro 1 - Escalas de medición de los constructos}

\begin{tabular}{|c|c|c|}
\hline Constructo / Ítem & Afirmación & Autores base \\
\hline \multicolumn{3}{|l|}{ Usabilidad } \\
\hline usab1 & Este sitio web es fácil de usar & $\begin{array}{l}\text { Kim, Xu y Koh (2004) } \\
\text { Flavian, Guinaliu y Gurrea (2006) }\end{array}$ \\
\hline usab2 & Este sitio web es fácil de entender & Flavian, Guinaliu y Gurrea (2006) \\
\hline usab3 & Es fácil encontrar la información que necesito en este sitio web & $\begin{array}{l}\text { Roy, Dewit y Aubert (2001) } \\
\text { Kim, Xu y Koh (2004) } \\
\text { Flavian, Guinaliu y Gurrea (2006) }\end{array}$ \\
\hline \multicolumn{3}{|l|}{ Satisfacción } \\
\hline sat1 & $\begin{array}{l}\text { Estoy satisfecho con las operaciones que he realizado en este } \\
\text { sitio Web }\end{array}$ & $\begin{array}{l}\text { Brockman (1998) } \\
\text { Sharma y Patterson (2000) } \\
\text { Veloutsou, Daskou y Daskou (2004). }\end{array}$ \\
\hline sat2 & $\begin{array}{l}\text { En términos generales, estoy satisfecho con el sistema de } \\
\text { funcionamiento de este sitio web }\end{array}$ & Brockman (1998) \\
\hline sat3 & Este sitio web ha satisfecho mis expectativas & Veloutsou, Daskou y Daskou (2004). \\
\hline \multicolumn{3}{|l|}{ Confianza } \\
\hline conf1 & $\begin{array}{l}\text { Realizar operaciones por este sitio web me genera una abso- } \\
\text { luta confianza }\end{array}$ & Doney y Cannon (1997) \\
\hline conf2 & Este sitio web cumple lo que promete & $\begin{array}{l}\text { Kumar, Scheer y Steenkamp (1995) } \\
\text { Roy, Dewit y Aubert (2001) }\end{array}$ \\
\hline conf3 & $\begin{array}{l}\text { Confío en que la operaciones que realizo a través de este sitio } \\
\text { web están libren de fallas }\end{array}$ & Roy, Dewit y Aubert (2001) \\
\hline
\end{tabular}

contactadas y un porcentaje de concreción de encuestas de un 4,6\%, se generó una muestra de 292 casos válidos. Los casos atípicos, las respuestas repetidas y los cuestionarios incompletos fueron controlados. El tamaño de la muestra cumple los requisitos de cantidad de encuestados necesarios para el tipo de análisis utilizado, con rangos entre 7 y 10 observaciones por cada ítem (HAIR y otros, 2005). En particular, la tasa de observaciones a ítems es de 32,4 para la muestra total.

En la Tabla 1 se detalla el perfil de la muestra, éste guarda relación con hallazgos previos que indican que quienes realizan transacciones por Internet son fundamentalmente hombres, personas de nivel educacional más elevado y de mayores ingresos (FLAVIAN, GUINALIU y TORRES, 2006).

\section{Dimensionalidad, Fiabilidad y Validez de las Escalas}

En primer lugar, para realizar el análisis de dimensionalidad de la muestra, se empleó el análisis factorial exploratorio (HAIR y otros, 2005). Este análisis arrojó tres factores, agrupados en confianza, satisfacción y usabilidad, con cargas factoriales adecuadas de cada ítem sobre su factor (menor valor $=0,788$ ). Los tres factores explicaban un 80,9\% del total de la varianza, mostrando además valores correctos en el indicador KMO y en el test de esfericidad de Bartlett. Con este proceso, se pudo confirmar la unidimensionalidad de cada una de las escalas de medida (HAIR y otros, 2005).

Se realizó además, un análisis factorial confirmatorio por medio del método de ecuaciones estructurales. Considerando las diferentes sub-escalas o variables latentes, se realizó un proceso de mejora por medio de una estrategia de desarrollo de modelo (HAIR y otros, 2005). Este proceso, consiste en eliminar aquellos indicadores que no contribuyen a lograr un buen ajuste del modelo propuesto, considerando los tres criterios propuestos por Jöreskog y Sörbom (1993). El primer criterio, aparta del análisis aquellos indicadores que tengan una débil condición de convergencia $(t>2,58$; 


\section{Tabla 1 - Perfil de la muestra}

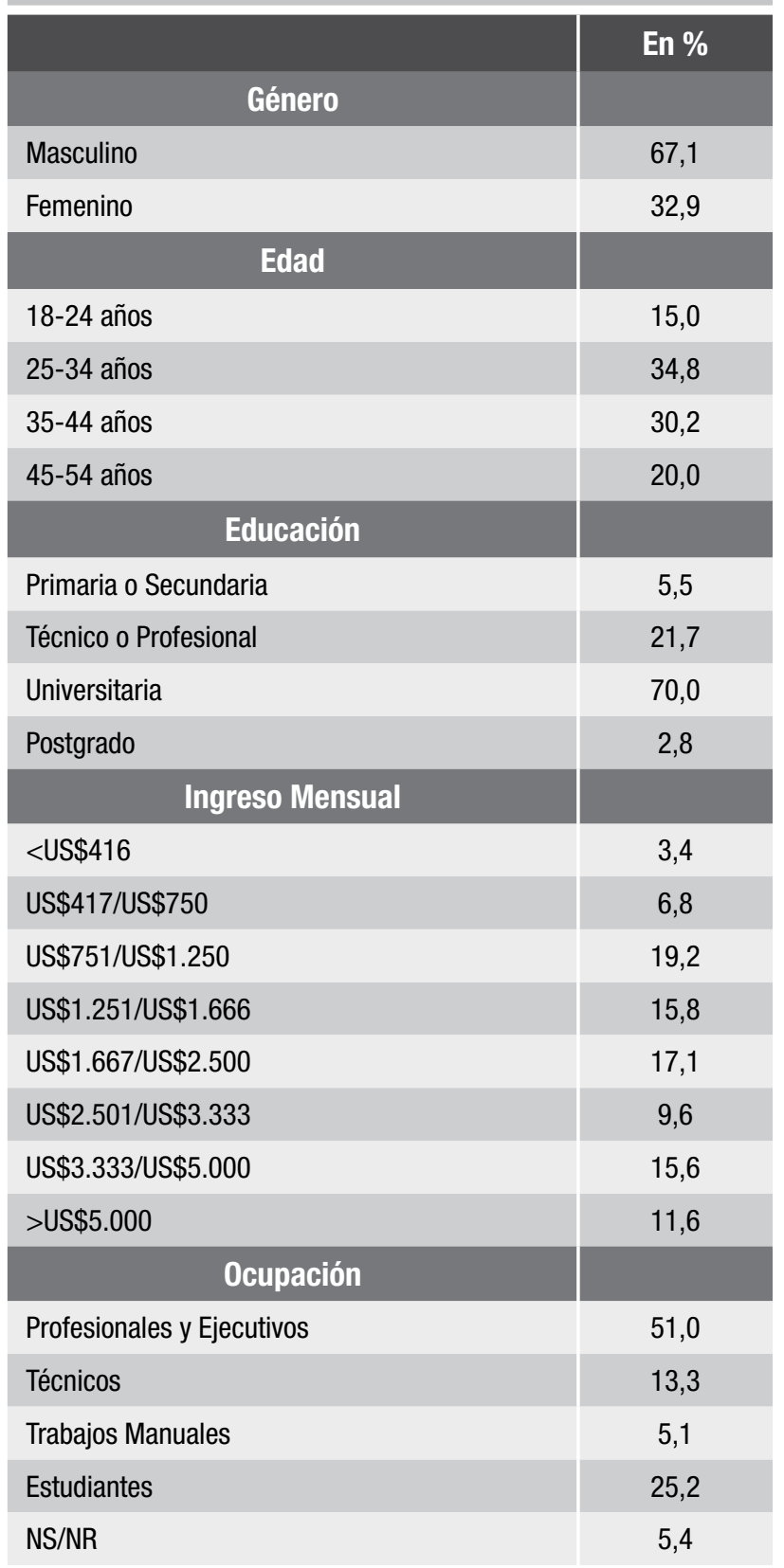

$\mathrm{p}=0,01)$. El segundo criterio, elimina aquellos indicadores que no son sustanciales $(\boldsymbol{\lambda}>0,5)$. Por último, el tercer criterio elimina aquellos indicadores que son menos explicados por el factor que los representa $\left(R^{2}>0,3\right)$.

Considerando estos criterios no fue necesario eliminar indicadores. Los ajustes del modelo resultaron satisfactorios: $\chi 2$ (g.l.) $=45,750(24) \mathrm{p} \leq 0,001$; NFI $=0,975$; IFI=0,988; CFI $=0,988$; GFI $=0,969$; RMSEA $=0,056$; Normed $\chi 2=1,90$.

Posteriormente, la fiabilidad de las escalas de medida, se comprobó por medio de tres test independientes: Alfa de Cronbach (CRONBACH, 1951), Fiabilidad Compuesta del Constructo (JÖRESKOG, 1971) y Análisis de la Varianza Extraída (AVE) (FORNELL y LARCKER, 1981). En todos los casos las escalas superaron los valores mínimos establecidos por estos test $(0,7 ; 0,7 ; 0,5$ respectivamente) y por lo tanto, se puede garantizar el buen grado de fiabilidad que tienen cada una de ellas (Tabla 2).

Finalmente, se analizó la validez convergente y la validez discriminante de los constructos. La validez convergente se confirmó al observar que en las tres escalas, los coeficientes estandarizados del modelo confirmatorio eran, en todos los casos, estadísticamente significativos al 0,01 y mayores a 0,5 (BAGOZZI y YI, 1988). Por su parte, la validez discriminante se confirmó a través de dos procedimientos: el test de diferencias Chi-cuadrado y el test del intervalo de confianza (BAGOZZI, 1981; ANDERSON y GERBING, 1988). El primero de ellos consiste en comparar la Chi-cuadrado entre el modelo resultante del análisis factorial confirmatorio y diferentes modelos alternativos en el que dos de sus dimensiones presentan una perfecta correlación. El segundo test, indica que existe validez discriminante entre dos variables latentes cuando en los intervalos de confianza resultantes de estimar la correlación entre ambas variables latentes no se encuentra incluido el valor 1,0. En todos los casos estudiados, se cumplieron las condiciones expuestas, pudiendo verificar ambos tipos de validez (Tabla 3).

Tabla 2 - Fiabilidad

\begin{tabular}{|l|c|c|c|}
\hline & Confianza & Satisfacción & Usabilidad \\
\hline Alfa de Cronbach & 0,83 & 0,92 & 0,89 \\
\hline Fiabilidad compuesta del constructo & 0,76 & 0,84 & 0,81 \\
\hline Análisis de la varianza extraída & 0,52 & 0,64 & 0,59 \\
\hline
\end{tabular}




\section{RESULTADOS}

La hipótesis planteada fue testeada utilizando un modelo de ecuaciones estructurales (BAGOZZI, 1981) y una estrategia de modelos rivales para contrastar el efecto directo versus el indirecto.

En primer lugar, los índices de ajuste del modelo principal, donde se consideran las relaciones usabilidad-satisfacción y satisfacción-confianza, superan ampliamente los valores exigidos (HAIR y otros, 2005): NFI $=0,975$; IFI $=0,988$; CFI $=0,988$; GFI $=0,968$; RMSEA $=0,054$; Normed $\chi 2=1,845$.

$\mathrm{Al}$ presentar el modelo coeficientes estandarizados significativos y mayores a cero, se puede afirmar que la usabilidad percibida afecta de manera positiva y directa a la satisfacción hacia los sitios web de subastas y, a su vez, la satisfacción afecta de manera positiva y directa a la confianza depositada por el usuario en este tipo de sitios web (Figura 1), siendo coherentes con lo expuesto en el marco teórico respecto de la determinación entre estas variables.

Posteriormente, a través del análisis de modelos rivales (BLOEMER y ODEKERKEN-SCHRODER, 2003) y siguiendo los requisitos propuestos por Morgan y Hunt (1994), se testearon los posibles modelos alternativos, con el fin de verificar que la satisfacción es una variable mediadora entre la usabilidad percibida y la confianza. En este caso, el modelo propuesto se comparó con dos modelos rivales posibles, que permiten comparar la acción directa e indirecta de la usabilidad sobre la confianza en el contexto de estudio (Figura 2).

En el primer caso, se consideró la influencia directa de la usabilidad percibida sobre la confianza (Modelo A). Los ajustes de este modelo alternativo resultaron considerablemente más deficientes ( $\mathrm{CFI}=0,898$; RMSEA $=0,159$; Normed $\chi 2=8,366)$ y la variable confianza fue explicada en un menor nivel $\left(R^{2}=0,42\right.$ versus $\mathrm{R}^{2}=0,38$ ).

En el segundo caso, se consideró una influencia de la usabilidad percibida sobre la confianza y sobre la satisfacción (Modelo B). Si bien los ajustes del modelo alternativo fueron iguales o levemente peores a los del modelo propuesto (CFI $=0,988$; RMSEA $=0,056$; Normed $\chi^{2}=1,899$ ), en este caso la relación usabilidad-confianza resultó no significativa, lo que permite concluir que el modelo propuesto en este estudio presenta una mejor parsimonia.

Lo anterior, sumado al hecho que existe un efecto indirecto significativo y positivo de la usabilidad sobre la confianza (coeficiente estandarizado de la relación indirecta $=0,46$ ), muestran que la satisfacción es un factor mediador clave en la relación entre estas dos variables, permitiendo corroborar lo propuesto en la hipótesis propuesta. Vale mencionar que el modelo estudiado cumple las condiciones de mediación propuestas por Baron y Kenny (1986), donde por una parte, la relación usabilidad-satisfacción es significativa al igual que la relación satisfacción-confianza, y por otra parte, una vez que estas relaciones están presentes en el modelo,

\section{Tabla 3 - Validez convergente y discriminante}

\begin{tabular}{|c|c|c|c|c|}
\hline \multicolumn{2}{|c|}{ Validez Convergente } & \multicolumn{3}{|c|}{ Validez Discriminante } \\
\hline Ítems & $\begin{array}{l}\text { Coeficientes } \\
\text { Estandarizados }\end{array}$ & Pares de Constructos & $\begin{array}{l}\text { Test de Diferencias } \\
\qquad 2 \text { (di) }\end{array}$ & $\begin{array}{l}\text { Intervalos de } \\
\text { Confianza }\end{array}$ \\
\hline Conf1 & $0,867^{*}$ & CONF-SAT & $211,140(1)^{\star}$ & 0,$565 ; 0,729$ \\
\hline Conf2 & $0,869^{*}$ & CONF-USAB & $271,694(1)^{*}$ & 0,$386 ; 0,594$ \\
\hline Conf3 & $0,639^{*}$ & SAT-USAB & $233,963(1)^{\star}$ & 0,$645 ; 0,781$ \\
\hline Sat1 & $0,878^{*}$ & & & \\
\hline Sat2 & $0,950^{*}$ & & & \\
\hline Sat3 & $0,827^{*}$ & & & \\
\hline Usab1 & $0,841^{*}$ & & & \\
\hline Usab2 & $0,925^{*}$ & & & \\
\hline Usab3 & $0,796^{\star}$ & & & \\
\hline
\end{tabular}

Nota: *Coeficientes significativos al 0.01 
la relación directa entre usabilidad y confianza presenta valores no significativos. Bajo éstas condiciones, la relación de mediación de la satisfacción puede ser clasificada como mediación solo-indirecta (ZHAO, LYNCH y CHEN, 2010).

\section{CONCLUSIÓN y DISCUSIÓN}

Considerando los resultados, se puede concluir que el principal aporte de este estudio está dado por el establecimiento de la mediación en una relación previamente discutida como directa en la literatura.

En particular, se comprueba empíricamente que el efecto de influencia de la usabilidad percibida sobre la confianza es indirecto y mediado por la satisfacción en el contexto de los sitios web de subastas. Este hecho se reafirma con el contraste de modelos rivales, donde se puede apreciar que: a) el efecto directo de la usabilidad sobre la confianza no es significativo, b) se generan como resultado modelos alternativos pobremente explicados y c) existe un efecto indirecto significativo entre la usabilidad y la confianza. Lo anterior, muestra un resultado distinto a lo habitualmente desarrollado en el estudio de la usabilidad y su relación con la confianza, siendo un resultado nuevo en la investigación de las relaciones online y más aún en el contexto de las subastas por
Internet, donde existe una falta de material teórico y empírico.

Además de lo anterior, es necesario mencionar, que las relaciones analizadas en este estudio se probaron en los sitios de subastas online, contexto en el que no se habían explorado anteriormente. Del mismo modo es posible destacar el hecho que se haya realizado en el contexto de un país Latinoamericano, donde existe escasa evidencia empírica en esta área de estudio.

Considerando los resultados presentados por este estudio, se podría desprender que para lograr la confianza de sus clientes, los administradores de los sitios de subastas online deberían poner especial atención en los factores que ayudan a percibir una mayor usabilidad de su sitio web de remates. En concreto, la gestión debería centrarse en disponer de un sistema con una estructura totalmente entendible por el usuario, con funcionalidades y contenidos disponibles y fáciles de encontrar. Además, el uso de las aplicaciones debe ser sencillo, incluso para usuarios nuevos, contando con formas simplificadas para llevar a cabo las ofertas y ventas dentro del sitio. Otras características deseables, incluyen que el sitio web funcione de manera rápida (tanto en la navegación, como en los sistemas de búsqueda internos), que el usuario tenga la capacidad de controlar todo lo que hace y, que en todo momento, tenga una noción clara de qué hacer y del lugar en que se encuentra dentro de la página web. Todo lo anterior, repercutirá en un incremento de la satisfacción, que a

\section{Figura 1 - Modelo estructural}

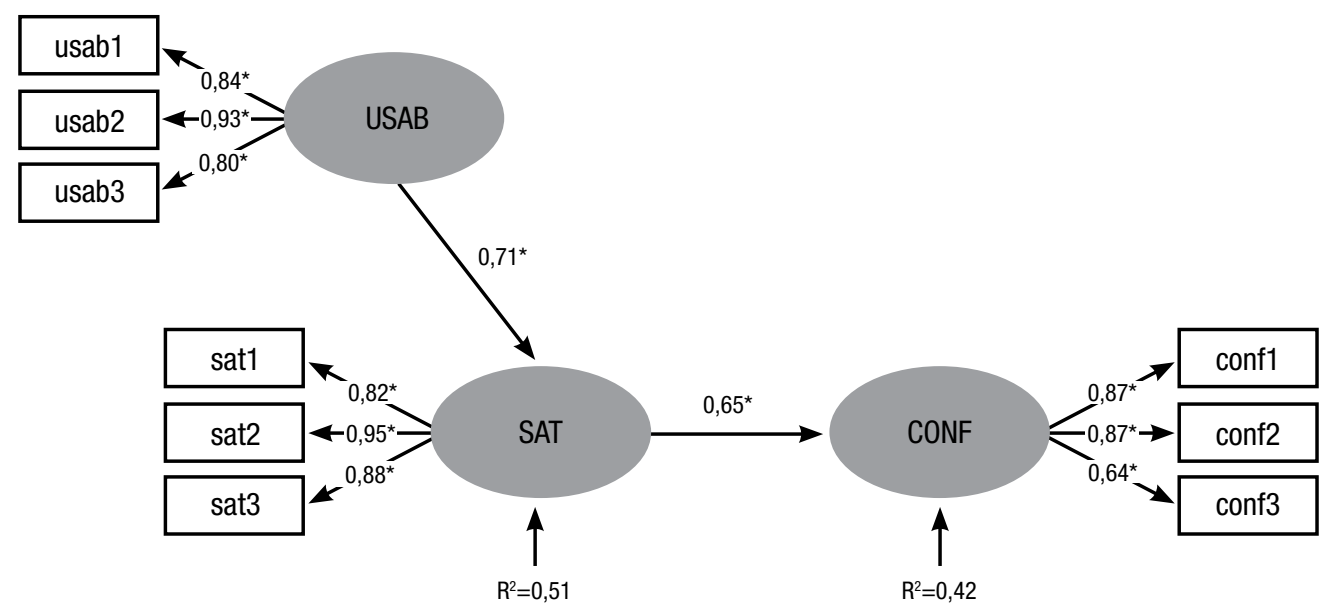

Nota: *Coeficientes significativos al 0.01 
su vez permitirá alcanzar un mayor nivel de confianza de los usuarios. Sin embargo, el efecto de la usabilidad estará diluido en su influencia sobre la satisfacción, debido a que existen otros componentes de la calidad del servicio que estarán actuando sobre ésta de una manera simultánea.

Asimismo, el presente estudio abre la puerta a la discusión y análisis de otros componentes de la calidad de servicio en ambientes online y su posterior influencia sobre importantes constructos del marketing relacional. Como antedicho, los componentes de la calidad del servicio (que pueden variar en un tipo de sitio web a otro), como la seguridad percibida, la exactitud y otros, debieran necesariamente tener una relación directa con la satisfacción, lo que genera espacios a futuras investigaciones para plantear y probar los caminos de determinación que nacen desde estas variables.

Las limitaciones de este estudio se enmarcan en el acotado marco de acción, que involucra una muestra en un determinado país (Chile) y en un determinado tipo de servicio web, sin embargo, como antedicho, no es el objetivo de esta investigación el ser exhaustivo, sino más bien, demostrar que las relaciones entre los constructos en los ambientes online están lejos de ser completamente cubiertas, y en especial que los factores clave deben ser estudiados inicialmente de manera aislada para posteriormente realizar generalizaciones, de manera de entender con la debida profundidad la naturaleza de las relaciones entre los constructos.

\section{REFERENCIAS}

AGARWAL, R; VENTKATESH, V. Assessing a firm's web presence: a heuristic evaluation procedure for the measurement of usability. Information Systems Research, v. 13, n. 2, p. 168-186, 2002.

\section{Figura 2 - Modelos rivales}

A)

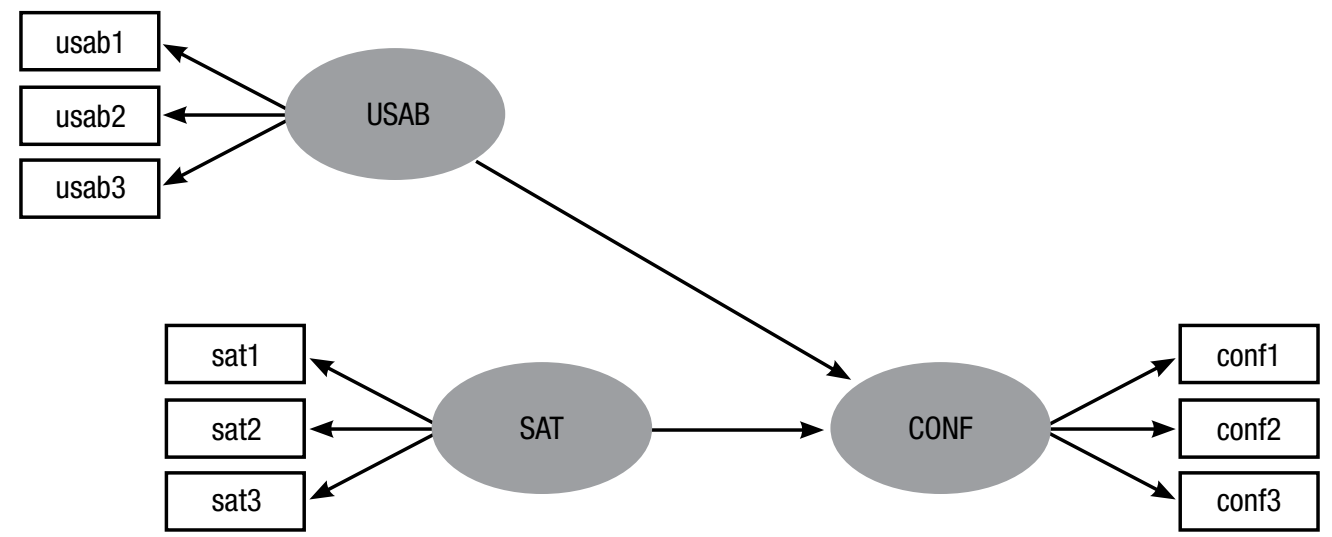

B)

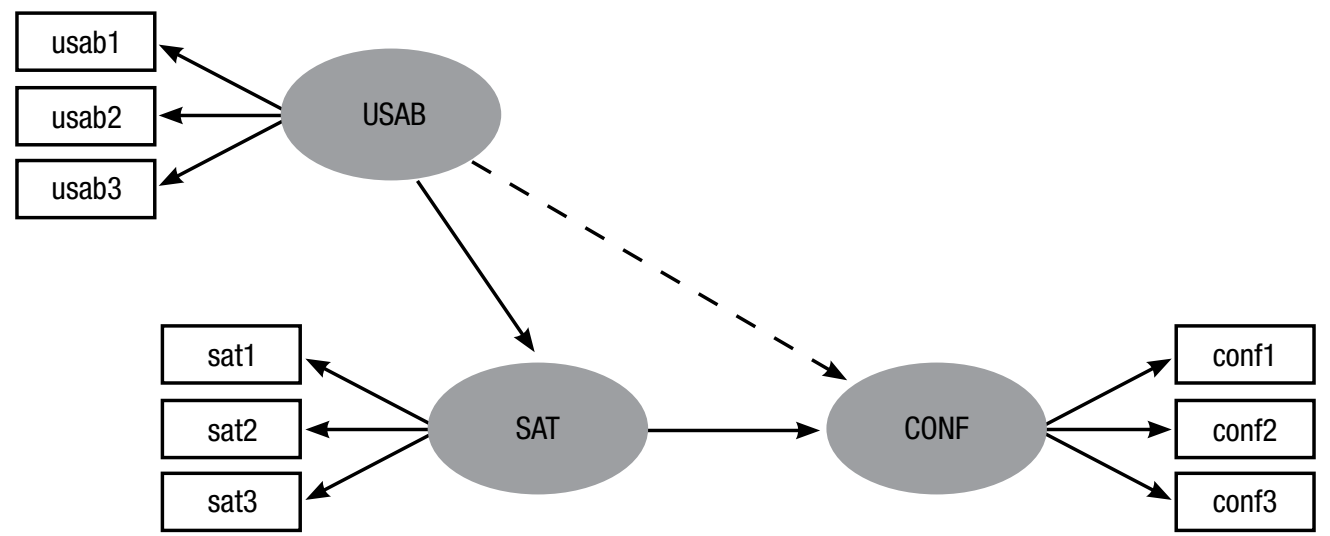


AGRIAWAN, A; THAKUR, R. A parsimonious model of the antecedents and consequences of online trust. Journal of Internet Commerce, v. 7, n. 1, p. 74-94, 2008.

ANDERSON, J.C; GERBING, D.W. Structural equation modeling in practice: a review and Recommended Two-Step Approach. Psychological Bulletin, v. 103, n. 3, p. 411-423, 1998.

ANTONY, S; Lin, Z; Xu, B. Determinants of escrow service adoption in consumer-to-consumer online auction market: an experimental study. Decision Support Systems, v. 42, n. 3, p. 1889-1900, 2006.

BAGOZZI, R.P. Evaluating structural equations models with unobservable variables and measurement error: a comment. Journal of Marketing Research, v. 18, n. 3, p. 375-381, 1981.

BAGOZZI, R.P; YI, Y. On the evaluation of structural equation models. Journal of the Academy of Marketing Science, v. 16, n. 1, p. 74-95, 1988.

BARNES, S.J; VIDGEN, T.R. An integrative approach to the assessment of e-commerce quality. Journal of Electronic Commerce Research, v. 3, n. 3, p. 114-127, 2002.

BARON, R.M; KENNY, D.A. The moderator-mediator variable distinction in social psychological research: conceptual, strategic, and statistical considerations. Journal of Personality and Social Psychology, v. 51, n. 6, p. 1173-1182, 1986.

BLOEMER, J; ODEKERKEN-SCHRODER, G. Antecedents and consequences of affective commitment. Australasian Marketing Journal, v. 11, n. 3, p. 33-43, 2003.

BROCKMAN, B.K. The influence of affective state on satisfaction ratings. Journal of Consumer Satisfaction, Dissatisfaction and Complaining Behavior, v. 11, p. 40-50, 1998.

CASALO, L.V; FLAVIAN, C; GUINALIU, M. The role of security, privacy, usability and reputation in the development of online banking. Online Information Review, v. 31, n. 5, p. 583-603, 2007.

CHEEMA, A; POPKOWSKI, P.T.L; BAGOZZI, R; COX, J; DHOLAKIA, U.M; GREENLEAF, E.A; PAZGAL, A; ROTHKOPF, M.H; SHEN, M; SUNDER, S; ZEITHAMMER, R. Economics, psychology, and social dynamics of consumer bidding in auctions. Marketing Letters, v. 16, n. 3/4, p. 401-413, 2005.
CRONBACH, L.J. Coefficient Alpha and the internal structure of test. Psychometrika, v. 16, n. 3, p. 297-334, 1951.

DE WULF, K; ODEKERKEN-SCHRODER, G. Assessing the impact of a retailer's relationship efforts on consumer' attitudes and behavior. Journal of Retailing and Consumer Services, v. 10, n. 2, p. 95-108, 2003.

DEWALLY, M; EDERINGTON, L. Reputation, certification, warranties, and information as remedies for seller-buyer information asymmetries: lessons from the online comic book market. Journal of Business, v. 79, n. 2, p. 693-729, 2006.

DIMITRIADES, Z.S. Customer satisfaction, loyalty and commitment in service organizations: some evidence from Greece. Management Research News, v. 29, n. 12, p. 782-800, 2006.

DONEY, P; CANNON, J.P. An examination of the nature of trust in buyer-seller relationships. Journal of Marketing, $\mathrm{v}$. 61, n. 2, p. 35-51, 1997.

EVANSCHITZKY, H; IYER, G.R; HESSE, J; AHLERT, D. E-satisfaction: a re-examination. Journal of Retailing, v. 80, n. 3, p. 239-247, 2004.

FAN, M; TAN, Y; WHINSTON, A.B. Evaluation and design of online cooperative feedback mechanism for reputation management. IEEE Transactions on Knowledge and Data Engineering, v. 17, n. 2, p. 244-254, 2005.

FLAVIAN, C; GUINALIU, M; GURREA, R. The influence of familiarity and usability on loyalty to online journalistic services: the role of user experience. Journal of Retailing and Consumer Services, v. 13, n. 5, p. 363-375, 2006.

FLAVIAN, C; GUINALIU, M; TORRES, E. How bricks-and-mortar attributes affect online banking adoption. International Journal of Bank Marketing, v. 24, n. 6, p. 406423, 2006.

FOGG, B; KAMEDA, T; BOYD, J; MARSHALL, J; SETHI, R; SOCKOL, M; TROWBRIDGE, T. Stanford-Makovsky web credibility study: investigating what makes web sites credible today. A Research Report by the Stanford Persuasive Technology Lab \& Makovsky \& Company, Stanford University, Disponible en: http://www.webcredibility.org, 2002.

FORNELL, C; LARCKER, D.F. Evaluating structural equation models with unobservable variables and measurement error. Journal of Marketing Research, v. 18, n. 1, p. 39-50, 1981. 
FU, L; SALVENDY, G. The contribution of apparent and inherent usability to a user's satisfaction in searching and browsing task on the web. Ergonomics, v. 45, n. 6, p. 415424, 2002.

GEFEN, D; KARAHANNA, E; STRAUB, D.W. Trust and TAM in online shopping: An integrated model. MIS Quarterly, v. 27, n. 1, p. 51-90, 2003.

GRAZIOLI, S; JARVENPAA, S.L. Perils of internet fraud: an empirical investigation of deception and trust with experienced internet. IEEE Transactions on Systems, Man and Cybernetics - Part A: Systems and Humans, v. 30, n. 4, p. 395-410, 2000.

HAIR, J.F; BLACK, W.C; BABIN, B; ANDERSON, R.E; TATHAM, R.L. Multivariate Data Analysis, London: Prentice-Hall International, 2005.

HU, X; LIN, A.B; WHINSTON, H; ZHANG, H. Hope or hype: on the viability of escrow services as trusted third parties in online auction environments. Information Systems Research, v. 15, n. 3, p. 236-249, 2004.

JONES, K; LEONARD, L.N.K. Trust in consumer-to consumer electronic commerce. Information \& Management, v. 45, n. 2, p. 88-95, 2008.

JÖRESKOG, K.G. Statistical analysis of sets of congeneric test. Psychometrika, v. 36, n. 2, p. 109-133. 1971.

JÖRESKOG, K.G; SORBOM, D. New feature in LISREL 8, Chicago: Scientific Software, 1993.

KIM, D.J; FERRIN, D.L; RAO, H.R. A study of the effect of consumer trust on consumer expectations and satisfaction: the Korean experience. En: Proceedings of the 5th Intl Conference on Electronic Commerce, ACM Press, New York, p. 310-315, 2003.

KIM, H; XU, Y; KOH, J. A comparison of online trust building between potential customers and repeat customers. Journal of Association for Information Systems, v. 5, n. 10, p. 392-420, 2004.

KIM, M.S; AHN, J.H. Management of trust in the e-marketplace. Journal of Information and Technology, v. 22, n. 2, p. 119-132, 2007.
KRACHER, B; CORRITONE, C.L; WIEDENBECK, S. A foundation for understanding online trust in electronic commerce. Information, Communication E Ethics in Society, v. 3, n. 3, p. 131-141, 2005.

KOUFARIS, M; HAMPTON-SOSA, W. The development of initial trust in an online company by new customers. Information \& Management, v. 41, n. 3, p. 377-397, 2004.

KUMAR, N; SCHEER, L.K; STEENKAMP, J.B.E. The effects of supplier fairness on vulnerable resellers. Journal of Marketing Research, v. 32, n. 1, p. 54-65, 1995.

LI, S; SRINIVASAN, K; SUN, B. Internet auction features as quality signals. Journal of Marketing, v. 73, n. 1, p. 7592, 2009.

LI, Y.M; YEH, Y.S. Increasing trust in mobile commerce through design aesthetics. Computers in Human Behavior, v. 26, n. 4, p. 673-684, 2010.

LICHTENSTEIN, D.R; NETEMEYER, R.G; BURTON, S. Distinguishing coupon proneness from value consciousness: an acquisition - transaction utility theory perspective, Journal of Marketing, v. 54, n. 3, p. 54-67, 1990.

LOONAM, M; O'LOUGHLIN, D. Exploring e-service quality: a study of Irish online banking. Marketing Intelligence E Planning, v. 26, n. 7, p. 759-780, 2008.

LUCKING-REILEY, D. Auctions on the Internet: what's being auctions, and how? Journal of Industrial Economics, v. 48, n. 3, p. 227-252, 2000.

MELNIK, M.I; ALM, J. Does a seller's ecommerce reputation matters? Evidence from eBay auctions. The Journal of Industrial Economics, v. 50, n. 3, p. 337-349, 2002.

MAYER, R.C; DAVIS, J.H; SHOORMAN, F.D. An integrative model of organizational trust. Academy of Management review, v. 20, n. 3, p. 709-734, 1995.

MORGAN, R.M; HUNT, S.D. The commitment-trust theory of relationship marketing. Journal of Marketing, v. 58, n. 3, p. 20-38, 1994.

NIELSEN, J. Designing Web Usability: The Practice of Simplicity, Indianapolis: New Riders Publishing, 2000. 
NIELSEN, J. Usability 101: Introduction to usability, 2003, disponible en: http://www.useit.com/alertbox/20030825. html. Acceso en: 15.05.2009.

OLIVER, R.L. Satisfaction; a Behavioral Perspective on the Consumer, New York: McGraw-Hill, 1997.

OLIVER, R.L. Whence consumer loyalty? Journal of Marketing, v. 63, n. 4, p. 33-44, 1999.

PALMER, J.W. Web site usability, design, and performance metrics. Information Systems Research, v. 13, n. 2, p. 151-167, 2002.

PAVLOU, P.A. Consumer acceptance of electronic commerce: integrating trust and risk with the technology acceptance model. International Journal of Electronic Commerce, v. 7, n. 3, p. 101-134, 2003.

PEARSON, J.M; PEARSON, A.M. An exploratory study into determining the relative importance of key criteria in web usability: A multi-criteria approach. Journal of Computer Information Systems, v. 48, n. 4, p. 115-127, 2003.

RAVALD, A; GRONROOS, C. The value concept and relationship marketing. European Journal of Marketing, v. 30, n.2, p. 19-30, 1996.

RESNICK, P; ZECKHAUSER, R; FRIEDMAN, E; KUWABARA, K. Reputation systems. Communications of the ACM, v. 43, n. 12 , p. $45-48,2000$.

ROY, M.C; DEWIT, O; AUBERT, B.A. The impact of interface usability on trust in web retailers. Internet Research, v. 11, n. 5, p. 388-398, 2001.

SANZO, M.J; SANTOS, M.L; VASQUES, R; ALVAREZ, L.I. The effect of market orientation on buyer-seller relationship satisfaction. Industrial Marketing Management, v. 32, n. 4, p. 327-345, 2003.

SELNES, F. An examination of the effect of product performance on brand reputation, satisfaction and loyalty. European Journal of Marketing, v. 27, n. 9, p. 19-35, 1993.

SHARMA, N; PATTERSON, P.G. Switching costs, alternative attractiveness and experience as moderators of relationship commitment in professional, consumer services. International Journal of Service Industry Management, v. 11, n. 5, p. 470-490, 2000.
SPILLER, P; LOHSE, G. A classification of the Internet retail stores. International Journal of Electronic Commerce, v. 2, n. 2, p. 29-56, 1998.

SZYMANSKI, D.M; HISE, R.T. E-satisfaction: an initial examination. Journal of Retailing, v. 76, n. 3, p. 309-322, 2000.

TUNG, F.C; LEE, M.S; CHEN, C.C; HSU, Y.S. An extension of financial cost and TAM model with IDT for exploring users' behavioral intentions to use the CRM information system. Social Behavior and Personality, v. 35, n. 5, p. 621-626, 2009.

URBAN, G.L; SULTAN, F; QUALLS, W.J. Placing trust at the center of your internet strategy. Sloan Management Review, v. 42, n. 1, p. 39-48, 2000.

VELOUTSOU, C; DASKOU, S; DASKOU A. Are the determinants of bank loyalty brand specific?. Journal of Financial Services Marketing, v. 9, n. 2, p. 113-125, 2004.

WALCZUCH, R; LUNDGREN, H. Psychological antecedents of institution-based consumer trust in e-retailing. Information \& Management, v. 42, n. 1, p. 159-177, 2004.

YANG, Z; FANG, X. Online service quality dimensions and their relationship with satisfaction. International Journal of Service Industry Management, v. 15, n. 3, p. 302-326, 2004.

ZAICHKOWSKY, J.L. Measuring the involvement construct, Journal of Consumer Research, v. 12, n. 4, p. 341-52, 1985.

ZBOJA, J.J; VOORHES, C.M. The impact of brand trust and satisfaction on retailer repurchase intentions. Journal of Services Marketing, v. 20, n. 5, p. 381-390, 2006.

ZHAO, X; LYNCH, J.G; CHEN, Q. Reconsidering Baron and Kenny: Myths and truths about mediation analysis. Journal of Consumer Research, v. 37, n. 2, p. 197-206, 2010.

ZVIRAN, M; GLEZER, C; AVNI, I. User satisfaction from commercial web sites: the effect of design and use. Information \& Management, v. 43, n. 2, p. 157-178, 2006. 